\title{
Smart homecare technologies for the visually impaired: recent advances
}

This article was published in the following Dove Press journal:

Smart Homecare Technology and TeleHealth

22 December 2014

Number of times this article has been viewed

Bruno Andò

Salvatore Baglio

Cristian Orazio Lombardo

Vincenzo Marletta

Department of Electric, Electronic and Informatics Engineering,

University of Catania, Catania, Italy
Correspondence: Bruno Andò

Department of Electric, Electronic and Informatics Engineering, University of Catania, Viale Andrea Doria 6, 95।25, Catania, Italy

Email bruno.ando@dieei.unict.it
Abstract: Accessibility and autonomy are fundamental needs for people with visual impairments wishing to enjoy their rights as citizens and to access buildings, premises, and other facilities. Dealing with the possibility of autonomously performing activities of daily living, two main aspects are mandatory: autonomous mobility and living independently. In particular, dealing with autonomous accessibility is strictly related to the possibility to perceive the environment and to gain a realistic and intuitive awareness of ongoing events and hazards. In order to promote living independently, caregivers (whether relatives or assistive services) need to be aware of the user's physical surroundings. The literature is rich in attempts to provide technical solutions to improve quality of life and autonomy for frail people, particularly with regard to the visually impaired. This review summarizes "state of the art" electronic aids for the visually impaired, and aims to to highlight the benefits and drawbacks of these aids.

Keywords: assistive systems, blindness, multisensor architecture, localization, activities of daily living

\section{Introduction}

Although some progress has been made, disability is one of the most relevant causes of social marginalization. In this paper, some issues regarding the visually impaired are discussed to highlight the dramatic impact of the "worst disability in the world" in terms of social marginalization, as stated by the World Health Organization. ${ }^{1}$

According to the most recent figures, there are hundreds of millions of people with major sight problems throughout the world. ${ }^{1}$ People with loss of sight face discrimination and infringement of their rights on a daily basis, be it at work, in trying to find work, or in going to a café, supermarket, or hospital.

The best way to help disabled people in the developing world is to provide them with autonomy. It is vital for blind and partially sighted people to have a good education and more autonomy to give them the skills needed to lead a full life. This is a new life concept, where the blind are not different but have only different abilities. ${ }^{1}$

For visually impaired people, accessibility and autonomy are fundamental needs because for an individual to enjoy his/her rights as a citizen, he/she should be able to access buildings, premises, and other facilities; an accessible environment means that a person will be able to seek employment, receive education and training, and pursue an active social and economic life.

The concept of autonomous accessibility is specifically related to the possibility of gaining a realistic and intuitive awareness of the environment as well as ongoing events 
and hazards. The advantages of tactile-based aids (tactile paths and tactile form of codification of information) are well-known, but there are some drawbacks with these solutions, including:

- social aspects of a tactile path, that could sometimes be interpreted as a mark of inferiority

- poor flexibility and adaptability of a tactile path, ie, modifications and reconfiguration of the environment requires rebuilding of the tactile path, which is expensive and time-consuming

- lack of information provided to the user in emergency circumstances and in the event of a critical situation

- difficulty with regard to a user being able to localize himself/herself inside the path and the impossibility of easily recovering the tactile path in the event that it is lost

- the use of arbitrary codification for exploitation of the tactile path, which is highly demanding

- use of hostile codification to access informative resources (such as notification of pictures, sculptures, flowers, plants, and other stimuli); in practice, the form of codification adopted is often highly demanding, while use of traditional tactile codification (eg, Braille) leads to exclusion of users who do not know the Braille codification.

The literature is replete with technical solutions aimed at improving quality of life and autonomy for frail people, particularly for the blind. ${ }^{2-6}$ The development of enabling information and communication technology, including both information and sensing technologies, has provided effective systems and devices to help frail people when performing their activities of daily living (ADL). Such solutions perform in the direction of guaranteeing the right to well-being and active ageing promoted all over the world and also by the Horizon 2020 policy. Two main aspects are addressed by research groups working in this field, ie, autonomous mobility and exploitation in indoor/outdoor environments and frail people being able to live independently. ${ }^{7-43}$

Another key point when dealing with solutions for ambient assisted living, especially for users with depressed sensorial function (eg, the visually impaired), is the development of convenient solutions to convey the information generated by assistive systems to users themselves. ${ }^{44,46-56}$

Changing the way in which the user is provided with information from their surroundings, from artificial coding to natural perception, is necessary to avoid masking of natural echoes (the user must always be able to "visualize" the environment), to improve user confidence in such technologies, and to reduce the amount of training required. ${ }^{5}$
In the following sections, a nonexhaustive but representative review of the state of the art in this field is given, against the efforts dedicated to strengthening these innovations both from the technological and methodological points of view. ${ }^{2-44,46-56}$ Since the aim of this review is to address assistive technologies, we do not discuss emerging topics in the field of bioengineering, like the artificial retina, or efforts to develop implantable prostheses able to partially restore vision to blind people.

The literature indicates that, despite good performance of electronic aids, some drawbacks have to be acknowledged, such as:

- high costs

- reliability issues

- poor quality of information provided to the user, in terms of quantity (redundancy) and continuity (existence of dead zones where the user is not assisted)

- inadequate user interfaces (eg, an acoustic interface masking natural echoes, or a vibratory interface conveying information that is poor or difficult to interpret); an interface must be for everyone and for everywhere, in that it should be easy to use and interpret, it should not mask natural echoes, and should be flexible and reconfigurable while conveying a suitable degree of information

- need for highly demanding training. ${ }^{6}$

The above-mentioned drawbacks lead to the visually impaired being diffident about using many of the available solutions. ${ }^{6}$ Similar consideration could be extended to other classes of perceptual impairment.

The development of new methodologies overcoming the above drawbacks, for example, by providing blind people with a realistic and intuitive perception of the environment, could solve some of the structural and communication barriers to their use in a concrete way. ${ }^{2,4,13,18,19,32,44} \mathrm{~A}$ realistic and intuitive perception of the environment is mandatory to address their real need of autonomy, to facilitate social inclusion, and to protect them against hazards.

An overview of solutions to assist blind users in mobility tasks is discussed in the next sections, along with innovative methodologies for the detection of ADL. The crucial task of providing the user with a convenient and natural form of information is introduced in the final section.

With the aim of illustrating frontier results produced by recent research activity, the main focus of this paper is on the methodologies adopted to implement smart signal processing rather than on an indepth description of the hardware involved. It should be taken into account these low cost solutions are able to guarantee system performances. 


\section{Assistive systems for autonomous mobility in the blind}

Assistive systems for frail people should be able to build awareness of the user's physical status within the environment and provide the user with optimized information allowing for safe and efficient exploitation of the environment. To this end, several technologies have been exploited, including ultrasonic, infrared, laser, and camera devices, while auditory and/or tactile solutions have been used to provide feedback relevant to the user. ${ }^{7-10}$

The use of a handheld device based on an ultrasonic beam to detect the distance between the user and obstacles and a vibrational-based user feedback system is exploited by the Miniguide. ${ }^{10}$ The possibility of adopting electromagnetic and optical systems to implement travel aids for blind people has been described..$^{11,12}$ The Kahru Tactile Outdoor Navigator ${ }^{13}$ is a wearable tactile harness-vest display that uses vibrating motors to provide directional navigation instructions to the user. Examples of devices exploiting a camera to perceive the environment and convenient translation of its contents into auditory or tactile perception include the vOICe system ${ }^{14}$ and Intelligent Glasses. ${ }^{15}$ Other solutions use digital tags, active badges, accelerometers, and other sensing systems to interact with the user once the user gets into the devices' range. ${ }^{16}$

Although notable efforts have been made to develop assistive systems with convenient performance, drawbacks have emerged, such as the discontinuous form of assistance provided to the user, high cost, and sometimes an arbitrary form of information conveyed to the user.

The main tasks required to implement an efficient and reliable assistive system for the autonomous mobility of frail people are summarized in Figure 1. The system must be able to locate the user, assess environmental hazards, and monitor user status. Moreover, a suitable decision-making system which produces the knowledge on the user-environment interaction is mandatory. Obviously, a suitable way to convey service and security information to the user is a critical feature of each assistive system. The main functionalities of an assistive system for the exploitation of indoor environments are now discussed.

\section{Absolute and relative indoor tracking systems}

Development of pedestrian tracking systems for indoor application is a challenge for researchers, especially for critical contexts. Two main approaches can be followed, ie, absolute tracking solutions and relative tracking systems. ${ }^{17-32}$

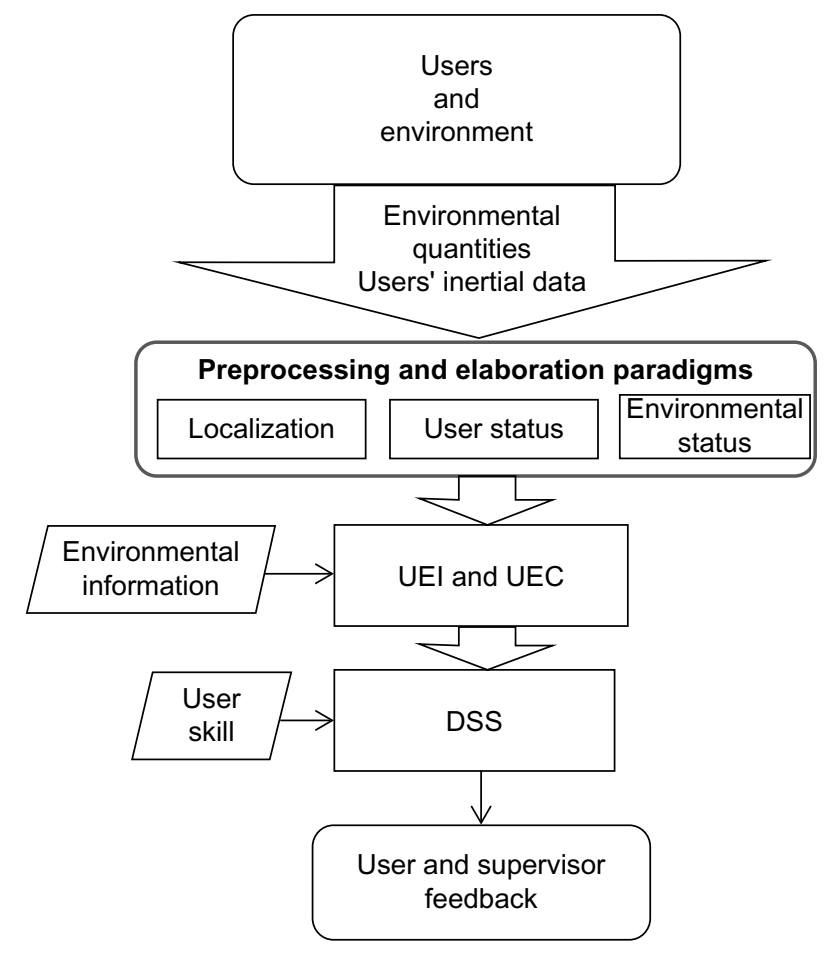

Figure I An approach to implement a smart assistive system for the autonomous mobility of blind people in indoor environments.

Abbreviations: DSS, decision support system; UEC, user environment contextualization; UEl, user environment interaction.

Concerning absolute indoor tracking, solutions based on wireless localization systems (such as Bluethooth, ZigBee, and radio-frequency identification) show an accuracy which is often not compatible with assistive purposes in an indoor environment. Moreover, drawbacks of such approaches include the high cost of their initial deployment and power budget. To overcome such drawbacks, a number of technologies have been proposed. As an example, the localization system adopted described by Andò et al ${ }^{17,18}$ is based on the continuous interaction between the user module, equipped with an ultrasound transmitter, and a distributed architecture of sensor nodes equipped with ultrasound receivers. This approach, which also exploits smart trilateration algorithms, allows for real time and very accurate estimation of the user's position within the environment. ${ }^{32}$ In order to provide the trilateration system with a high degree of redundancy, reinforcing the robustness and the robustness against faults of the system, more than three fixed reference points are usually adopted, as shown in Figure 2. ${ }^{32}$ As a first step, the algorithm proposed by Andò et a $1^{17,32}$ solves the trilateration problem locally, thus obtaining $M$ estimations of the user's position. The paradigm then performs a statistical analysis of the results to filter least probable solutions and finally processes the remaining results to calculate the optimal estimation. The multitrilateration 


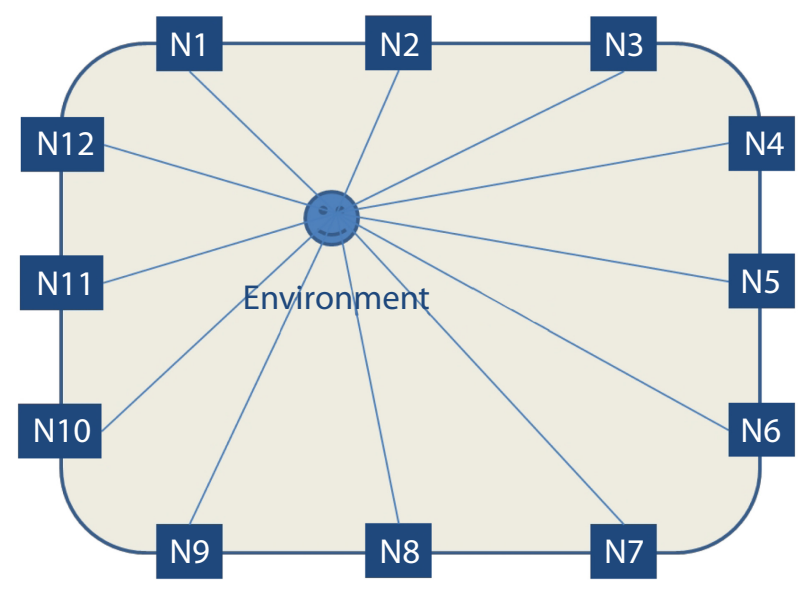

Figure 2 A two-dimensional trilateration approach with redundant sensor nodes $(\mathrm{N})$.

system ensures a suitable accuracy to the user localization task (in the order of centimeters), as well as robustness (especially when some sensor nodes fail). ${ }^{17,32}$

Despite their good performance, absolute indoor tracking systems require structured environments; ${ }^{19}$ inertial tracking can be adopted especially for remote monitoring of frail people in unstructured environments. Such a solution can provide accurate relative positioning information both in indoor and outdoor environments. Although these systems typically suffer for cumulative drift errors, both in walking distance and heading estimations, such drifts can be fixed through a periodic reset by absolute references. ${ }^{20}$

Advances in this context could come from the use of suitable data fusion techniques, often based on extended Kalman filters, ${ }^{21-23}$ aimed to improve performance of the navigation system. ${ }^{20}$

Concerning technologies, different solutions have been proposed, eg, standalone customized devices, ${ }^{24-26}$ smartphone-based platforms, ${ }^{27,28}$ and mixed systems. ${ }^{29,30}$

Although smartphone-based assistive systems could discourage frail people, considering that the cultural heritage in information and communication technology systems is rapidly spreading, such solutions can be very convenient for the safe and efficient exploitation of educational/cultural/job environments.

In line with the above considerations, our group has developed a fully smartphone-based tracking system for ambient assisted living-oriented architecture. ${ }^{31}$

In order to provide the user with a reliable walking path reconstruction, the approach schematized in Figure 3 aims to reduce the effect of environmental influences on the system estimations. The main feature of this solution resides in the possibility of providing a reliable heading estimation

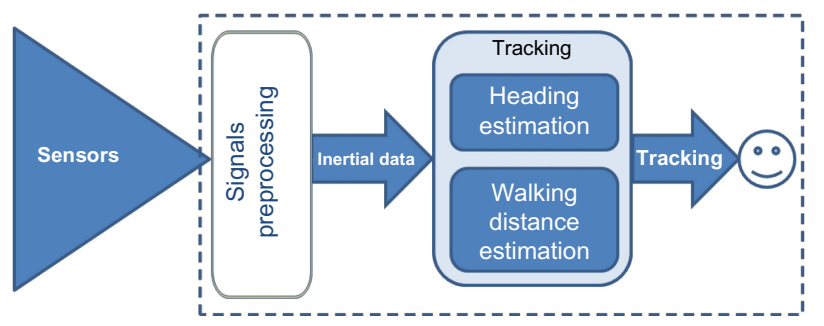

Figure 3 Methodology proposed for implementation of a reliable user tracking system designed to reduce the effect of environmental influences on path reconstruction estimation.

also in the presence of serious environmental influence (eg, magnetic field distortion), by exploiting constrained Kalman filters. Moreover, for the walking distance estimation adaptive models, exploiting information on user inertial dynamics, are proposed..$^{31}$

\section{Building user/environment awareness}

An example of a solution to provide blind users with a suitable form and amount of information for safe and efficient living in indoor environments is described in Andò et al. ${ }^{17,32}$ The main idea addressed in this work is to define a paradigm to be used when the user and the environment must be mutually aware of each other. The user can be any entity needing to interact with the environment and needing to have context awareness, such as persons, robots, or moving machines. The cognitive sensor network proposed by Andò et al $\mathrm{a}^{17,32}$ aims to perceive the user within the environment and to monitor the environment itself. Consequently, the cognitive system can gain an awareness of the user-environment interaction, thus providing the user with effective assistance to exploit the environment.

To implement this type of cognitive task, a smart distributed sensor network installed in the environment is used along with a user module. Obviously, a fundamental role is accomplished by paradigms aimed to build awareness of the user-environment interaction. ${ }^{17,32}$

Our RESIMA project ${ }^{17,18}$ aims to develop paradigms to assist blind users in indoor environments. The system, which is schematized in Figure 1, is based on a wireless sensor network and an advanced form of signal processing that performs several tasks, such as localization of the user, estimation of user inertia, and monitoring of strategic environment quantities.

An ultrasound-based localization system has been used to perform real time localization of the user with an accuracy of $\sim 4 \mathrm{~cm}$. This localization system exploits an optimization procedure to fix problems caused by a rough installation of the sensor nodes. An example of a user path reconstruction is given in Figure 4. ${ }^{32}$ The information acquired is then conveyed 
to the user-environment interaction and user-environment contextualization tools which represent the first level of the data integration aimed to improve the user awareness of physical status in respect to the environment.

The user-environment interaction tool is designed to perceive the user's interaction with obstacles and services available in the environment. As schematized in Figure 5, this task is accomplished by exploiting knowledge of the user's position and a map of the environment. The userenvironment contextualization tool builds information on anomalous and dangerous events, due to users' failures or hazards in the environment. To this end, the user-environment contextualization tool analyzes the user's status as respect to their status in the environment.

Information generated by the user-environment interaction and user-environment contextualization tools are then exploited by a decision support system. The decision support system provides information to the user for a safe and effective site exploitation and to the supervisor to manage hazards or specific user requests. The possibility of providing the user with an effective form of assistance represents the main advantage of the RESIMA system against currently available solutions. ${ }^{17,18}$

\section{Smart sensing solutions to detect ADL}

ADL includes a wide set of actions characterizing people's habits especially in their personal living spaces. Monitoring of ADL can be considered mandatory for frail

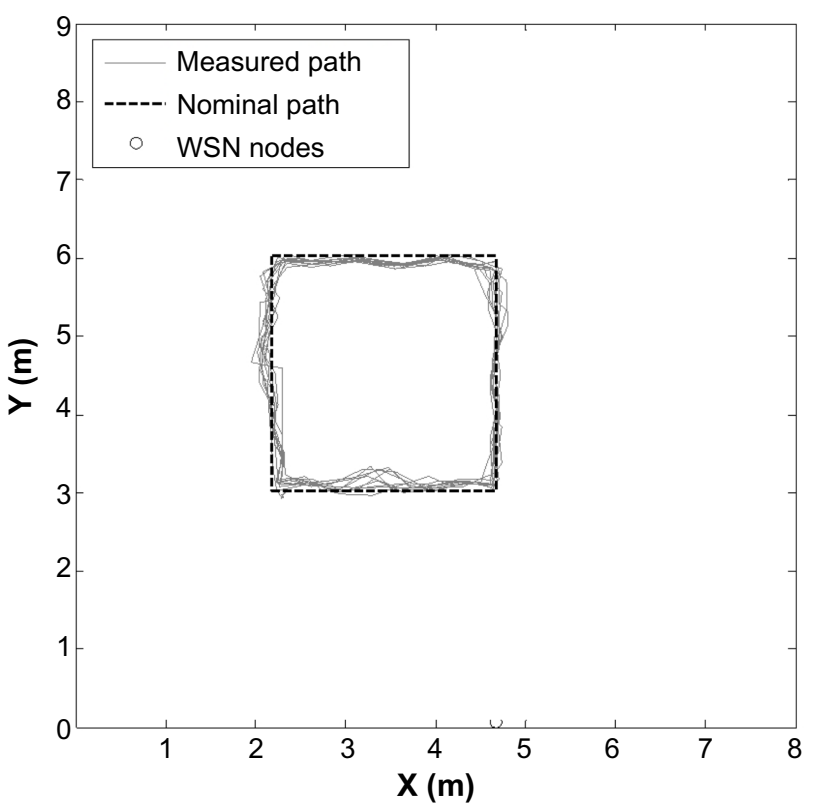

Figure 4 Example of results obtained by the trilateration algorithm. Abbreviation: WSN, wireless sensor networks.

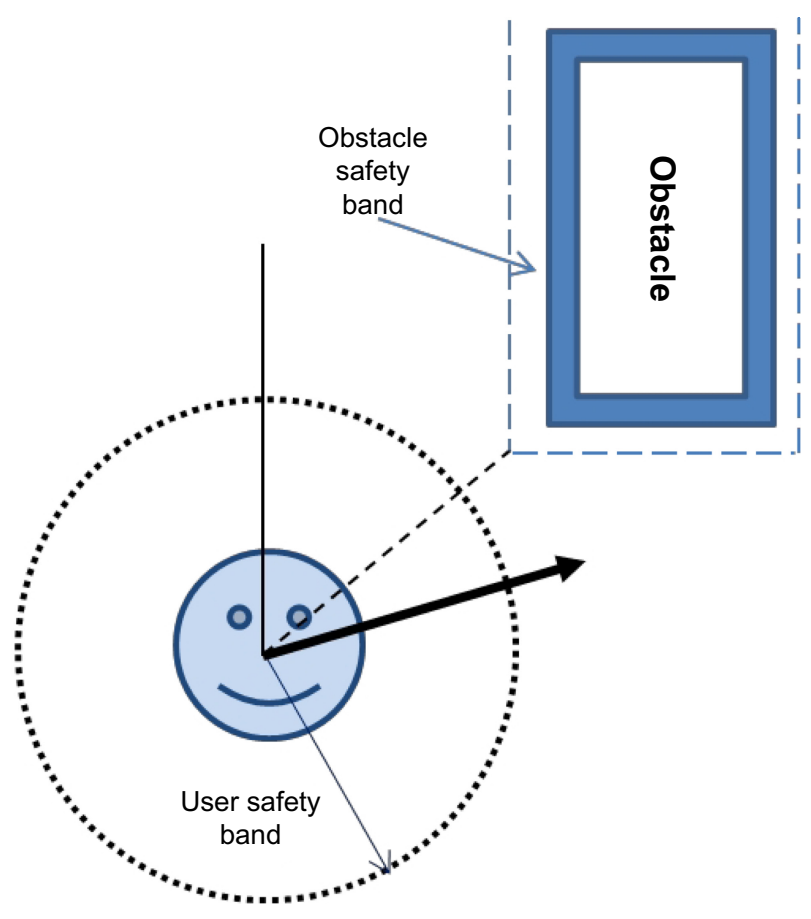

Figure 5 Schematization of the approach adopted to implement user-environment interaction functionality. Two safety areas, around the user and the obstacle, are defined to improve the performance of the assistive system in providing the user with alert notifications.

people, in order to guarantee effective and safe independent living. ${ }^{33}$ Negotiation of stairs, user posture, falls, sitting, standing up, and lying down are common activities to be detected. In falls in particular cause a lot of injuries among frail people.

The availability of reliable and user-friendly solutions for monitoring of ADL would be very useful for gaining a real time awareness of the user's condition and to provide the required assistance. From a technological point of view, both customized devices ${ }^{34-36}$ and smartphone-based platforms have been proposed in the literature for ADL monitoring.

Although smartphones are not always readily accepted by frail people due to the skill required to operate these devices, ADL detectors based on such a platform do not require user interaction and the smartphone can be conveniently positioned on the user's body. Conversely, some of the features of smartphones like automatic dialing and powerful signal processing are very strategic for the implementation of ADL monitoring. 37,40

Dunkel et $\mathrm{al}^{37}$ have addressed the use of smartphones and complex processing paradigms for downfall detection. A smartphone-based architecture for monitoring of human physical activities and its application to assist frail people is described in Franco et al; $;^{38}$ the possibility to estimate trunk position during bipedal stance in remote rehabilitation contexts, by exploiting signals from a gyroscope and an accelerometer embedded in a smartphone, is addressed by 
the same authors. ${ }^{38}$ Investigation of human falls based on the inertial sensing feature of smartphones is presented by Tacconi et al. ${ }^{27}$ A smartphone-based fall detection system, using threshold algorithms to process user acceleration and heading information is described by Quang Viet et al. ${ }^{40}$ A paradigm for interpretation of body movement exploiting a built-in triaccelerometer in smartphones is presented in $\mathrm{He}$ et $\mathrm{al}^{41}$, Fahim et al have proposed ${ }^{42}$ a solution to the problem of independent living by frail users, which exploits sensors deployed in the environment (on different objects) and the communication, storage, and processing features of smartphones supporting cloud-based signal processing.

The smartphone-based platform addressed by Andò et a ${ }^{43}$ is aimed at providing effective solutions for ADL detection in ambient assisted living contexts. The main task of the assistive system proposed is to acquire awareness of common ADL, such as avoidance of falls, negotiation of stair, and sitting, in order to provide frail users with a suitable degree of assistance. Advantages of the system developed are the use of a fully smartphone-based solution, the hip position of the device, as well as the possibility to recognize falls from other ADLs. However, the advantage of the proposed approach is the possibility to separate prevention of falls from other ADL and to classify among different kind of falls. The former is a relevant task in the remote monitoring of impaired people, while the latter is mandatory when implementing diagnostic or rehabilitation tasks. The strategy adopted to detect ADL exploits the cross-correlation between the body acceleration and ADL signatures. This approach makes the system robust against external influences. Moreover, two classification algorithms are proposed to process the extracted features (crosscorrelation values). The first algorithm exploits a threshold mechanism while the other is based on principal component analysis. In order to implement an adaptive threshold definition mechanism for different application contexts, the system sensibility and specificity have been taken into account. In order to test the system, simulated intentional actions were performed by healthy subjects. ${ }^{43}$ The subjects were asked to wear the system on their right hip and to perform a sequence of tasks. As discussed by Andò et al, ${ }^{43}$ the results obtained highlight the good sensitivity of the proposed methodology along with a reasonable specificity. The latter assures a negligible amount of false positive notifications, especially in discriminating falls from other activities. This platform could be exploited in both indoor and outdoor contexts, such as living environments, museums, hospitals, and public sites as well as for monitoring of patients recently discharged by the hospital.

\section{Providing the user with an efficient form of information}

The way assistive systems provide the information to blind people is key to improving their self-confidence and autonomy. Examples in the state of the art reveal that the information is often codified in arbitrary forms, by tactile or auditory perception. Efforts in developing nonvisual forms of presentation are also described. ${ }^{44}$ Gibson (1966) defined haptics as "the sensibility of the individual to the world adjacent to his body by use of his body" ${ }^{45}$ Our opinion is that haptic user interfaces play a fundamental role in the framework of assistive systems for the visually impaired. A state of the art survey on haptics is available in Varalakshmi et al, ${ }^{46}$ while a comprehensive review of haptic methods for data visualization is given in Panëels and Roberts. ${ }^{47} \mathrm{~A}$ few examples of interesting results are briefly discussed here.

Moustakas et $\mathrm{al}^{48}$ have proposed a force-field haptic rendering method for converting videos of three-dimensional maps into haptic data. An haptic interface for orientation and mobility training of blind users is proposed in Schloerb et al. ${ }^{49} \mathrm{~A}$ haptic system for the visually impaired exploiting a smartphone platform is presented in Akhter et al. ${ }^{50} \mathrm{~A}$ solution allowing the visually impaired to remotely identify objects of interest and to navigate through natural environments is proposed in Horvath et al. ${ }^{51}$ Other haptic solutions to assist impaired users have also been reported, ${ }^{52-54}$ and also applications dedicated to children. ${ }^{55}$

The development of a haptic cane has been described by Andò et al. ${ }^{5,56}$ The device exploits a tactile interface to provide the user with information on imminent obstacles and their position within the environment. The system basically consists of a short cane with an embedded ultrasound sensing strategy to perceive the position of an obstacle and an active handle. ${ }^{5,56}$ The handle implements a natural haptic codification strategy, which conveys to the user's palm a sensation similar to the one provided by a traditional long white cane.

The results of a study by Andò and Ascia ${ }^{5}$ was dedicated to understand the sensation provided on the user's hand palm by a traditional white cane bumping against obstacles. It has been observed that different kinds of bumps produce different hand palm solicitations. The haptic cane, proposed $i^{5}$ exploits such map of correspondence to reproduce into the user hand palm sensations similar to those perceived by a white cane. This functionality has been implemented by a distributed actuation system embedded on the cane handle.

The main advantage of the proposed solution is being able to provide the user with a natural form of codification regarding the position of a detected obstacle, rather than an arbitrary form of codification. This methodology will be 
valuable from a social point of view, giving autonomy to blind people performing mobility tasks. In particular, the user can achieve information on the environment without a physical interaction with objects and other people.

\section{Disclosure}

The authors report no conflicts of interest in this work.

\section{References}

1. World Health Organization. Visual impairment and blindness. Available from: http://www.who.int/mediacentre/factsheets/fs282/en/. Accessed October 12, 2014.

2. Bujacz M, Baranski P, Moranski M, Strumillo P, Materka A. Remote mobility and navigation aid for the visually disabled. In: Sharkey PM, Lopes-dos-Santos P, Weiss PL, Brooks AL, editors. Proceedings of the Seventh Conference on Disability, Virtual Reality and Associated Technologies with ArtAbilitation. Maia, Portugal, September 8-11, 2008.

3. Velázquez R. Wearable assistive devices for the blind. In: Lay-Ekuakille A, Mukhopadhyay SC, editors. Wearable and Autonomous Biomedical Devices and Systems for Smart Environment: Issues and Characterization. Lecture Notes in Electrical Engineering Series Volume 75. Berlin, Germany: Springer; 2010.

4. Farcy R, Bellik Y. Comparison of various interface modalities for a locomotion assistance device. In: Miesenberger K, Klaus J, Zagler W, editors. Computers Helping People with Special Needs. Berlin, Germany: Springer; 2002.

5. Andò B, Ascia A. Navigation aids for the visually impaired: from artificial codification to natural sensing. IEEE Instrum Meas Mag. 2007;10:44-51.

6. Andò B, Baglio S, La Malfa S, Marletta V. Innovative smart sensing solutions for the visually impaired. In: Pereira J, editor. Handbook of Research on Personal Autonomy Technologies and Disability Informatics. Hershey, PA, USA: Medical Information Science Reference; 2011.

7. Andò B, Graziani S. Multisensor strategies to assist blind people: a clear-path indicator. IEEE Trans Instrum Meas. 2009;58: 2488-2494.

8. Andò B. A smart multisensor approach to assist blind people in specific urban navigation tasks. IEEE Trans Neural Syst Rehabil Eng. 2008;16: 592-594.

9. Kay L. A sonar aid to enhance spatial perception of the blind: engineering design and evaluation. Radio and Electronic Engineer. 1974;44:605-627.

10. The Miniguide mobility aid [webpage on the Internet]. Adelaide: GDP Research. Available from http://www.gdp-research.com.au/minig_1. htm. Accessed November 13, 2014.

11. Villanueva J, Farcy R. Optical device indicating a safe free path to blind people. IEEE Trans Instrum Meas. 2012;61:170-177.

12. Scalise L, Primiani VM, Russo P, et al. Experimental investigation of electromagnetic obstacle detection for visually impaired users: a comparison with ultrasonic sensing. IEEE Trans Instrum Meas. 2012;61: 3047-3057.

13. Gemperle F, Ota N, Siewiorek D. Design of a wearable tactile display. Presented at the Fifth International Symposium on Wearable Computers, October 8-9, 2001, Zurich, Switzerland.

14. Meijer P. An experimental system for auditory image representations. IEEE Trans Biomed Eng. 1992;39:112-121.

15. Velazquez R, Fontaine E, Pissaloux E. Coding the environment in tactile maps for real-time guidance of the visually impaired. Presented at the IEEE International Symposium on Micro-NanoMechatronics and Human Science, November 5-8, 2006, Nagoya, Japan.

16. Andò B. Sensors that provide security for people with depressed receptors. IEEE Mag Instrum Meas. 2006;9:58-63.
17. Andò B, Baglio S, Lombardo CO, Marletta V, Pergolizzi EA, Pistorio A. (2013) RESIMA: A New WSN Based Paradigm to Assist Weak People in Indoor Environment. IEEE International Workshop on Measurements and Networking Proceedings (M\&N); 7-8 October, 2013; Napoli. 206-209.

18. Andò B, Baglio S, Lombardo C. RESIMA: an assistive paradigm to support weak people in indoor environments. IEEE Trans Instrum Meas. 2014;63:2522-2528

19. Andò B, Savalli N. CANBUS networked sensors use in orientation tools for the visually impaired wired versus wireless technology. IEEE Trans Instrum Meas. 2008;11:49-52.

20. Chen W, Chen R, Chen Y, Kuusniemi H, Wang J. An effective Pedestrian Dead Reckoning algorithm using a unified heading error model. Position Location and Navigation Symposium, 2010. Available from: http:// ieeexplore.ieee.org/xpl/abstractAuthors.jsp?tp=\&arnumber $=550730$ 0\&url=http $\% 3 \mathrm{~A} \% 2 \mathrm{~F} \% 2$ Fieeexplore.ieee.org\%2Fiel5\%2F5492941\% 2F5507125\%2F05507300.pdf\%3Farnumber\%3D5507300. Accessed October 11, 2014.

21. Kaniewski P, Kazubek J. Integrated system for heading determination. Acta Phys Pol A. 2009;116:325-330.

22. Yan L, Qiang W, Junbin L, Wark T. MCMC-based indoor localization with a smart phone and sparse WiFi access points. Presented at the Annual IEEE International Conference on Pervasive Computing and Communications, March 19-23, 2012, Lugano, Switzerland.

23. Kim JW, Jang HJ, Hwang DH, Park C. A step, stride and heading determination for the pedestrian navigation system. Journal of Global Positioning Systems. 2004;3:273-279.

24. Goyal P, Ribeiro VJ, Saran H, Kumar A. Strap-down Pedestrian DeadReckoning system. Presented at the 2011 International Conference on Indoor Positioning and Indoor Navigation, September 21-23, 2011, Guimaraes, Portugal.

25. Chi-Chung L, Chen-Pin C, Yu-Chee T, Sheng-An C, Lun-Chia K. A Walking Velocity Update technique for pedestrian dead-reckoning applications. Available from: http://people.cs.nctu.edu.tw/ yctseng/papers.pub/sensor58.walking-update-pimrc-2011.pdf. Accessed October 12, 2014.

26. Jimenez AR, Seco F, Prieto JC, Guevara J. Indoor pedestrian navigation using an INS/EKF framework for yaw drift reduction and a foot-mounted IMU. Presented at the Seventh Workshop on Positioning Navigation and Communication, March 11-12, 2010, Dresden, Germany.

27. Tacconi C, Mellone S, Chiari L. Smartphone-based applications for investigating falls and mobility. Presented at the Fifth International Conference on Pervasive Computing Technologies for Healthcare, May 23-26, 2011, Dublin, Ireland.

28. Trehard G, Boukallel M, Lamy-Perbal S. Indoor pedestrian localisation solution based on anemometry sensor integration with a smartphone. Presented at the 2012 International Conference Indoor Positioning and Indoor Navigation, November 13-15, 2012, Montbeliard, France.

29. Gadeke T, Schmid J, Zahnlecker M, Stork W, Muller-Glaser KD. Smartphone pedestrian navigation by foot-IMU sensor fusion. Presented at the Conference on Ubiquitous Positioning, Indoor Navigation, and Location Based Service, October 3-4, 2012, Helsinki, Finland.

30. Wakuda Y, Asano S, Koshizuka N, Sakamura K. Ubiquitous sensorbased pedestrian dead-reckoning for LBS applications. Presented at the 2012 International Symposium on Micro-NanoMechatronics and Human Science, November 4-7, 2012, Nagoya, Japan.

31. Andò B, Baglio S, Lombardo CO, Marletta V. An advanced tracking solution fully based on native sensing features of smartphone. Presented at the IEEE Sensors Applications Symposium, February 18-20, 2014.

32. Andò B, Baglio S, La Malfa S, Marletta V. A sensing architecture for mutual user-environment awareness case of study: a mobility aid for the visually impaired. IEEE Sensors Journal. 2011;11-3:634-640.

33. Suryadevara NK, Mukhopadhyay SC. Wireless sensor network based home monitoring system for wellness determination of elderly. IEEE Sensors Journal. 2012;12-6:1965-1972.

34. Rescio G, Leone A, Siciliano P. Supervised expert system for wearable MEMS accelerometer-based fall detector. Journal of Sensors. 2013; 2013:1-11. 
35. Panahandeh G, Mohammadiha N, Leijon A, Handel P. Chest-mounted inertial measurement unit for pedestrian motion classification using continuous hidden Markov model. Presented at the 2012 IEEE International Instrumentation and Measurement Technology Conference, November 16-21, 2012, New Orleans, LA, USA.

36. Kang JM, Yoo T, Kim HC. A wrist-worn integrated health monitoring instrument with a tele-reporting device for telemedicine and telecare. IEEE Trans Instrum Meas. 2006;55:1655-1661.

37. Dunkel J, Bruns R, Stipkovic S. Event-based smartphone sensor processing for ambient assisted living. Presented at the IEEE Eleventh International Symposium on Autonomous Decentralized Systems, March 6-8, 2013, Mexico City, Mexico.

38. Franco C, Fleury A, Gumery PY, Diot B, Demongeot J, Vuillerme N. iBalance-ABF: a smartphone-based audio-biofeedback balance system. IEEE Trans Biomed Eng. 2013;60:211-215.

39. Ketabdar H, Lyra M. System and methodology for using mobile phones in live remote monitoring of physical activities. Presented at the 2010 IEEE International Symposium on Technology and Society, June 7-9, 2010, Wollongong, Australia.

40. Quang Viet V, Lee G, Choi D. Fall detection based on movement and smart phone technology. Presented at the 2012 IEEE International Conference on Computing and Communication Technologies, Research, Innovation, and Vision for the Future, February 27, 2012 to March 1, 2012, Ho Chi Minh City, Vietnam.

41. HeY, LiY, Bao S-D. Fall detection by built-in tri-accelerometer of smartphone. Presented at the 2012 IEEE-EMBS International Conference on Biomedical and Health Informatics, January 2-7, 2012, Hong Kong, People's Republic of China.

42. Fahim M, Fatima I, Sungyoung L, Young-Koo L. Daily life activity tracking application for smart homes using android smartphone. Presented at the 14th International Conference on Advanced Communication Technology, February 19-22, 2012, Phoenix Park, South Korea.

43. Andò B, Baglio S, Lombardo CO, Marletta V, Pergolizzi EA, Pistorio A. An event polarized paradigm for ADL detection in AAL context. Presented at the 2014 IEEE International Instrumentation and Measurement Technology Conference, May 12-14, 2014, Montevideo, Uruguay.

44. Loftin RB. Multisensory perception: beyond the visual in visualization. Computing in Science and Engineering. 2003;5:56-58.
45. Gibson JJ. The senses considered as perceptual systems. Boston: Houghton Mifflin; 1966.

46. Varalakshmi BD, Thriveni J, Venugopal KR, Patnaik LM. Haptics: state of the art survey. International Journal of Computer Science Issues. 2012;9:234-244.

47. Panëels S, Roberts JC. Review of designs for haptic data visualization. IEEE Trans Haptics. 2010;3:119-137.

48. Moustakas K, Nikolakis G, Kostopoulos K, Tzovaras D, Strintzis MG. Haptic rendering of visual data for the visually impaired. IEEE MultiMedia. 2007;14:62-72.

49. Schloerb DW, Lahav O, Desloge JG, Srinivasan MA. BlindAid: virtual environment system for self-reliant trip planning and orientation and mobility training. Paper presented at the 2010 IEEE Haptics Symposium, March 20-26, 2010, Waltham, MA, USA.

50. Akhter S, Mirsalahuddin J, Marquina FB, Islam S, Sareen S. A smartphone-based haptic vision substitution system for the blind. Presented at the IEEE 37th Annual Northeast Bioengineering Conference, April 1-3, 2011, Troy, NY, USA.

51. Horvath S, Galeotti J, Bing W, Klatzky R, Siegel M, Stetten G. FingerSight: Fingertip haptic sensing of the visual environment. IEEE J Transl Eng Health Med. 2014;2:1-9.

52. Hayward V, Astley OR, Cruz-Hernandez M, Grant D, Robles-DeLa-Torre G. Haptic interfaces and devices. Sensor Review. 2004;24: 16-29.

53. Cassidy B, Cockton G, Coventry L. A haptic ATM interface to assist visually impaired users. Presented at the 15th International ACM SIGACCESS Conference on Computers and Accessibility, October 21-23, 2013, Bellevue, WA, USA.

54. Yoshino K, Shinoda H. Contactless touch interface supporting blind touch interaction by aerial tactile stimulation. Presented at the 2014 IEEE Haptics Symposium, February 20-23, 2014, Houston, TX, USA.

55. Patomäki S, Raisamo R, Salo J, Pasto V, Hippula A. Experiences on haptic interfaces for visually impaired young children. Presented at the Sixth International Conference on Multimodal Interfaces, October 13-15, 2004, State College, PA, USA.

56. Andò B, Baglio S, Pitrone N. A contactless haptic cane for blind people. Presented at the 12th IMEKO TC1-TC7 Joint Symposium on Man, Science and Measurement, September 3-5, 2008, Annecy, France.
Smart Homecare Technology and TeleHealth

\section{Publish your work in this journal}

Smart Homecare Technology and TeleHealth is an international, peer-reviewed, open access online journal publishing original research, reviews, editorials and commentaries on the application of technology to support people and patients at home and in assisted living centers to optimize healthcare and management resources. Specific topics in the journal include: Development and application of

\section{Dovepress}

devices within the home and embedded in appliances; Healthcare provider communication and education tools; and drug ordering and adherence. The manuscript management system is completely online and includes a very quick and fair peer-review system, which is all easy to use. Visit http://www.dovepress.com/ testimonials.php to read real quotes from published authors. 